\title{
Role of debulking surgery in combination with immune therapy: A successfully treated case of locally advanced mucosal melanoma
}

\author{
TAKAHIRO KIMURA ${ }^{1}$, TAKAYUKI TAKAHAMA ${ }^{2}$, TOMOKO WAKASA $^{3}$, SHIORI ADACHI $^{1}$, \\ YUSAKU AKASHI ${ }^{2}$, TAKAO TAMURA ${ }^{2}$ and KATSUNARI YANE ${ }^{1}$ \\ Departments of ${ }^{1}$ Otorhinolaryngology, ${ }^{2}$ Medical Oncology and ${ }^{3}$ Diagnostic Pathology, \\ Kindai University Nara Hospital, Ikoma, Nara 630-0293, Japan
}

Received December 21, 2020; Accepted August 23, 2021

DOI: $10.3892 / \operatorname{mco} .2021 .2435$

\begin{abstract}
Immune checkpoint inhibitors (ICIs) have markedly changed the treatment landscape for melanoma; however, their efficacy and applications are currently limited and medical requirements remain unmet. The present case study reports on a 85-year-old female patient who visited our outpatient clinic with a 1-month history of a buccal mucosa mass and was diagnosed with locally advanced mucosal melanoma of the head and neck. The patient's tumor progressed right after the administration of nivolumab, compromising oral intake. Palliative debulking surgery was performed. Subsequently, the other part of the melanoma on the hard palate slightly decreased in size without forming new lesions for more than one year after surgery. The present case exemplifies that tumor volume reduction surgery may increase the response to ICI and may prolong the duration of response. This combination therapy may be more effective in patients whose tumors increase in size after administration of ICIs or whose tumor is already large at the beginning of treatment. The combination of ICIs and debulking surgery may become an important treatment option in the future for locally advanced mucosal melanoma.
\end{abstract}

\section{Introduction}

Malignant melanoma is the result of cancerous transformation of melanin pigment cells. The molecular mechanisms of carcinogenesis and progression of malignant melanoma have been reported to include cyclin-dependent kinase (CDK) inhibitor 2A deletion, NRAS and BRAF mutations, and activation of melanocyte inducing transcription factor and CDK2 (1). Therefore, it is essential to develop therapeutic strategies using these molecular targets. Combination therapy with BRAF

Correspondence to: Dr Takayuki Takahama, Department of Medical Oncology, Kindai University Nara Hospital, 1248-1 Otoda, Ikoma, Nara 630-0293, Japan

E-mail: takahama_t@med.kindai.ac.jp

Key words: debulking surgery, immune checkpoint inhibitor, combination therapy, nivolumab, mucosal melanoma and MEK inhibitors is already in clinical use for malignant melanoma with BRAF V600E mutation (2). In addition, the US Food and Drug Administration approved the combination of dabrafenib and trametinib for unresectable or metastatic malignant melanoma in January 2014 (2).

By contrast, the standard of care for malignant melanoma without specific genetic mutations has been systemic chemotherapy. Dacarbazine has long been the systemic chemotherapy of choice for metastatic or inoperable malignant melanoma. However, the response rate to dacarbazine as a single agent is $\sim 10 \%$ and the complete response rate is $<5 \%$ (3). Various multidrug combinations, including dacarbazine, have been devised, but none of them have achieved a survival benefit. Hence, the development of novel treatment strategies is highly encouraged. ICIs have markedly improved treatment outcomes for melanoma (4). Prolonged progression-free survival (PFS) has been observed with nivolumab monotherapy, and furthermore, combination therapy with ipilimumab led to improvements in PFS more than ipilimumab monotherapy (5); thus, nivolumab monotherapy and combination with ipilimumab are considered one of the standards of care as first-line therapies for advanced melanoma today. However, as the patients experience more adverse events, combination therapy is difficult to be adapted to elderly patients. Treatment for elderly patients with melanoma tends to be difficult, as the response rate to nivolumab monotherapy is only $20-40 \%(6,7)$. The present study reported on an elderly case of locally advanced mucosal melanoma in the head and neck that was successfully treated with nivolumab and resection of one of the tumors that progressively grew despite nivolumab administration.

\section{Case report}

Case presentation. In July 2019, an 85-year-old female patient visited Kindai University Nara Hospital (Ikoma, Japan) with a 1-month history of a buccal mucosa mass. The patient had no particular complications or diseases under treatment and no oral medications. Initial physical findings included conjoined black area in the hard palate and gums and a protruding hemorrhagic lesion in the right buccal mucosa (Fig. 1A and B). No cervical lymph node metastasis or distant metastases were present. Serum lactate dehydrogenase was 148 U/1 (normal range, 129-221 U/1). Biopsies of the hard palate and buccal 
mucosal lesions were performed and both of them were diagnosed as malignant melanoma. The mutation status of BRAF was investigated with the Cobas ${ }^{\circledR} 4800$ BRAF V600 Mutation Test (Roche Diagnostics) and the result was negative.

Treatment. The tumor of the patient was deemed unresectable. Due to its location in the oral cavity, functions such as mastication and deglutition would not be preserved after resection. Considering the patient's age and adverse events, nivolumab monotherapy was administered instead of ipilimumab + nivolumab combination therapy. The patient received nivolumab (Ono Pharma) $240 \mathrm{mg}$ in total every two weeks. After two months, the tumor in the buccal mucosa had grown larger and bled, compromising oral intake (Fig. 2A). The tumor in the buccal mucosa did not invade the skin of the cheek; therefore, it appeared that the skin of the cheek was able to be preserved after tumor resection. During nivolumab treatment, the already present tumor exhibited enlargement. Of note, the growing oral mass impaired the patient's oral intake ability and bleeding from the tumor became more prominent. These conditions impacted the patient's quality of life and as the tumor hemorrhage was uncontrolable, palliative local treatment was deemed necessary. Palliative buccal mucosa tumor resection was thus performed.

The right buccal mucosal tumor was resected under local anesthesia. Macroscopically, resection was possible without residual tumor tissues by partially resecting the buccinator muscle (Fig. 2B). The resected specimen was examined pathologically and the sample exhibited malignant melanoma growth (Fig. 3A). There were no findings suggestive of pseudoprogression, such as infiltration of immune cells, so-called tumor-infiltrating lymphocytes (8). Almost all tumor cells were viable on histological examination and there was no necrosis, apoptosis or infarction. A certain amount of CD45-positive lymphocytes were present in the submucosal layer, but inside the tumor nodule, no lymphocytes were present (Fig. 3B).

The wound had healed 1 month postoperatively, leaving only a mild cicatricial contracture and no trismus. Nivolumab therapy was re-administered after exacerbation and was able to be continued for $>5$ months without any discontinuation due to adverse events. The buccal mucosal tumor did not recur and the black lesion on the hard palate mildly decreased in size without the formation of any new lesions. Eventually, the patient was able to eat unobstructedly without any tumor bleeding (Fig. 4A and B).

Immunohistochemical analysis. The resected tissues were fixed with phosphate-buffered $10 \%$ neutral formalin and processed routinely for paraffin-embedded tissue sections and with $\mathrm{H} \& \mathrm{E}$ staining.

Immunohistochemical staining for Leukocyte Common Antigen (CD45) was performed with the Histofine Histostainer 36A (Nichirei Biosciences, Inc.) using primary antibodies against CD45 (cat. no. 422071; clone no. PD7/26,2B11; pre-diluted working solution for Histostainer; Nichirei Biosciences, Inc.) according to manufacturer's protocol.

\section{Discussion}

Mucosal melanoma of the head and neck is more aggressive than melanoma of the skin (9). Complete resection with adequate margins is required for good outcomes; however, there are numerous important organs in the head and neck region and therefore, it is difficult to perform resection with adequate margins, resulting in a variety of functional disorders (9). Until recently, chemotherapy with dacarbazine was the main first-line therapy for unresectable melanomas. The overall response rate of dacarbazine was only $13.4 \%$ and the median survival duration ranged from 5.6 to 11 months (3). Thus, the response rate of treatment with dacarbazine is low and treatment efficacy is limited. The situation has changed drastically with the advent of ICIs. Today, ipilimumab + nivolumab combination therapy and nivolumab monotherapy are the standard of care as first-line therapies (10). The results of a pooled analysis of trials of immune checkpoint inhibitors in malignant mucosal melanoma have indicated that, while combination therapy is more likely to result in a high response, it is also associated with a higher frequency of serious adverse events leading to treatment discontinuation (7). As combination therapy has an increased incidence of adverse events, nivolumab monotherapy is thought to be an appropriate treatment option for elderly patients, such as the present case. However, good outcomes may not be achieved in over half of the patients, as the response rate of nivolumab monotherapy is only $20-40 \%(6,7)$.

Several factors, such as PD-L1 status or tumor burden, have been proposed as predictors of the efficacy of nivolumab $(6,10)$. In the present case, surgical debulking may have influenced the lasting effectiveness of nivolumab by two factors. The first is the reduction of the tumor burden and the second is the abscopal effect associated with the surgery. In ICI therapy, better therapeutic outcomes have been achieved when the ratio of activated T-cells to tumor burden was increased (11). In other words, a higher tumor burden would exhaust activated T-cells, which may prevent a better therapeutic response. Debulking surgery increased the number of activated T-cells, thereby contributing to a better clinical response. It was also reported that the tumor burden was related to the efficacy of ICIs in the treatment of small-cell lung cancer; they also noted that larger tumor volumes tended to have more robust T-regulatory cell infiltration, which induces immune tolerance (12). Furthermore, baseline tumor size is a prognosticator during treatment with pembrolizumab for melanoma (13).

The abscopal effect is frequently reported as a distant anti-tumor effect in radiotherapy with an immune checkpoint inhibitor. Regulating the tumor microenvironment or cancer antigen release may cause a cancer immune response at distant sites (14). Oronsky et al (15) reported an abscopal effect in patients with unresectable cancers treated by tumor reduction surgery or resection of metastatic lesions undergoing PD-L1 inhibitor therapy. It may also be inferred that surgical maneuvers are able to lead to release of cancer antigens, which mobilizes effector T-cells to generate the abscopal effect. This suggests that reducing systemic tumor burden or large resection of masses may be expected to enhance the effect of ICIs or lead to the abscopal effect.

The application of ICIs for post-operative adjuvant therapy of melanomas has been established. Pembrolizumab or nivolumab were reported as an effective post-operative adjuvant therapy for stage III advanced melanoma (16-18). These results suggested that tumor load reduction and post-operative adjuvant chemotherapy may prolong PFS. 


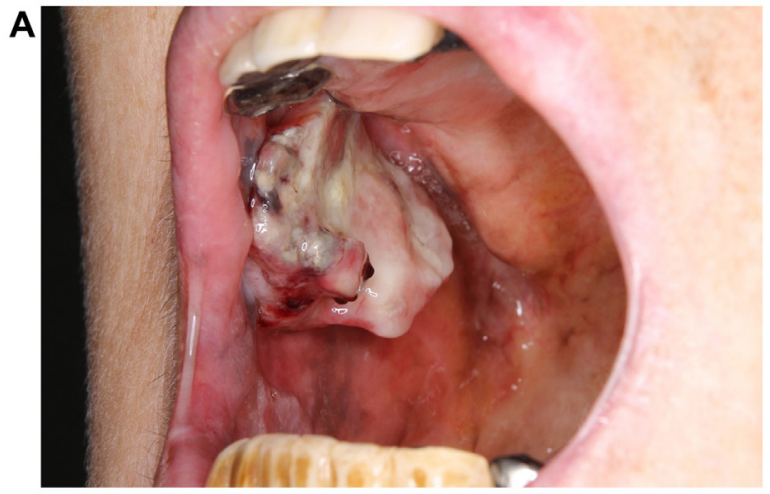

B

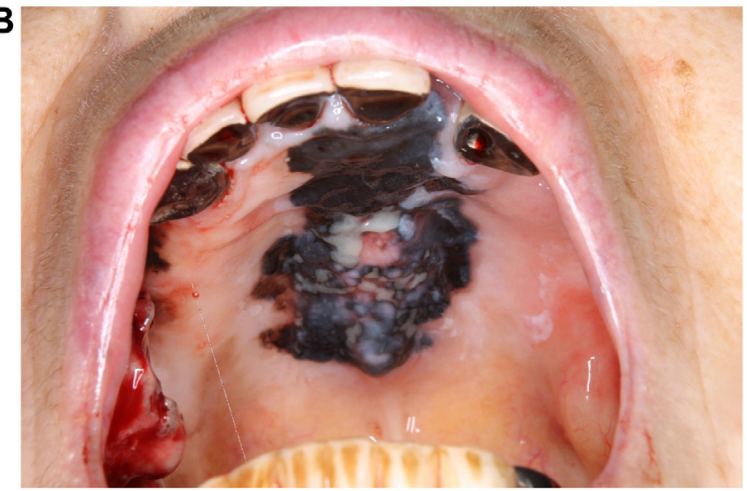

Figure 1. Images from the initial physical examination. (A) A hemorrhagic protruding lesion was present in the right buccal mucosa. (B) Black area in the hard palate.
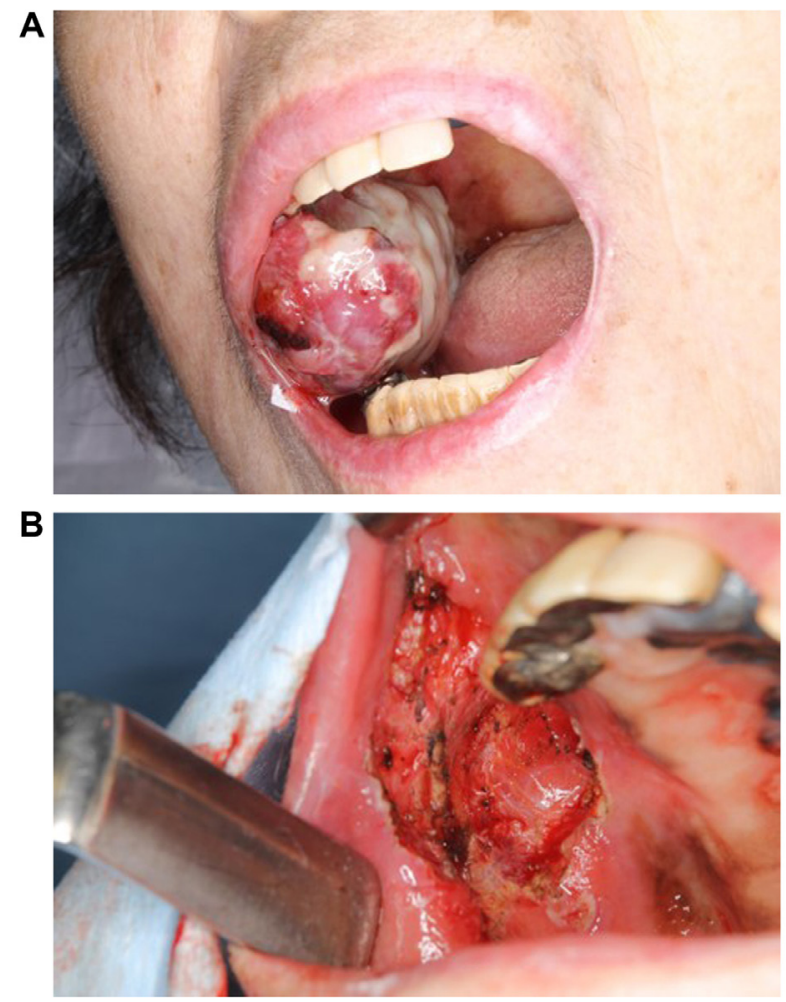

Figure 2. Macrophotographs of the buccal mucosal lesion at the time of mass enlargement and after resection. (A) Oral lesion two months after nivolumab administration. The tumor in the buccal mucosa was enlarged compared to the lesions before the start of treatment. The hemorrhagic tumor compromised oral intake. (B) Post-surgical photo. The right buccal tumor and part of the buccinator muscle were resected with a horizontal margin of $5 \mathrm{~mm}$. Fibrin glue was scattered on the cut buccal surface to prevent post-operative pain and bleeding. A peripheral black spot lesion remained.
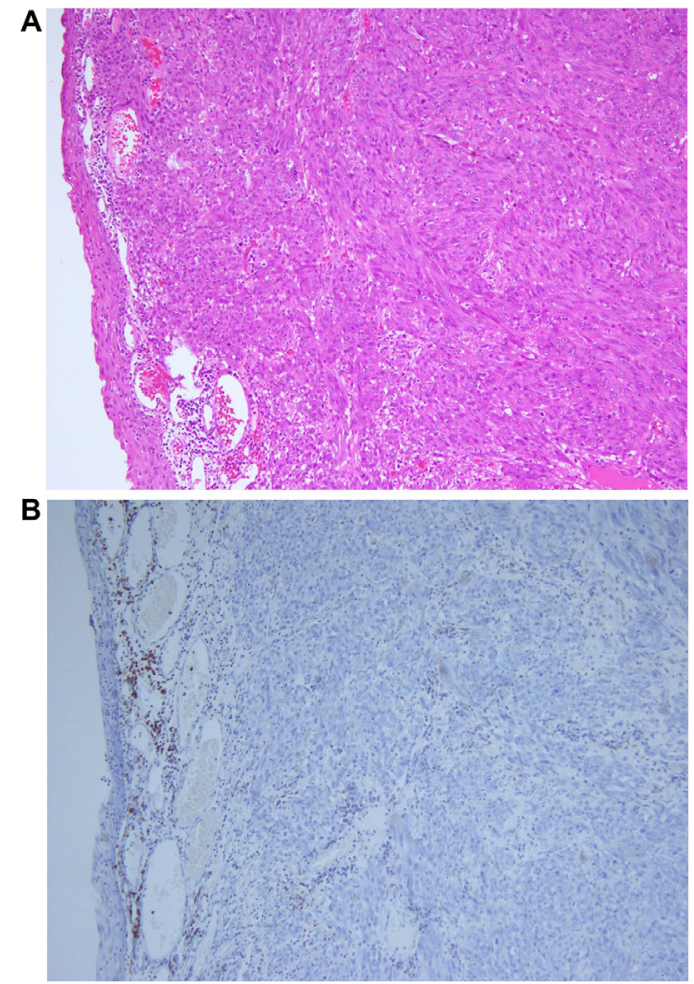

Figure 3. Histological findings of resected mucosal melanoma. (A) Macroscopic findings (H\&E staining). Almost all tumor cells were viable and there was no necrosis, apoptosis or infarction. (B) Macroscopic findings (immunohistochemical staining for CD45). CD45R-positive lymphocytes were present in the submucosal layer (brown staining), but not inside the tumor nodule. This observation suggests that no lymphocyte infiltration of the tumor occurred (magnification, $\mathrm{x} 100$ ).
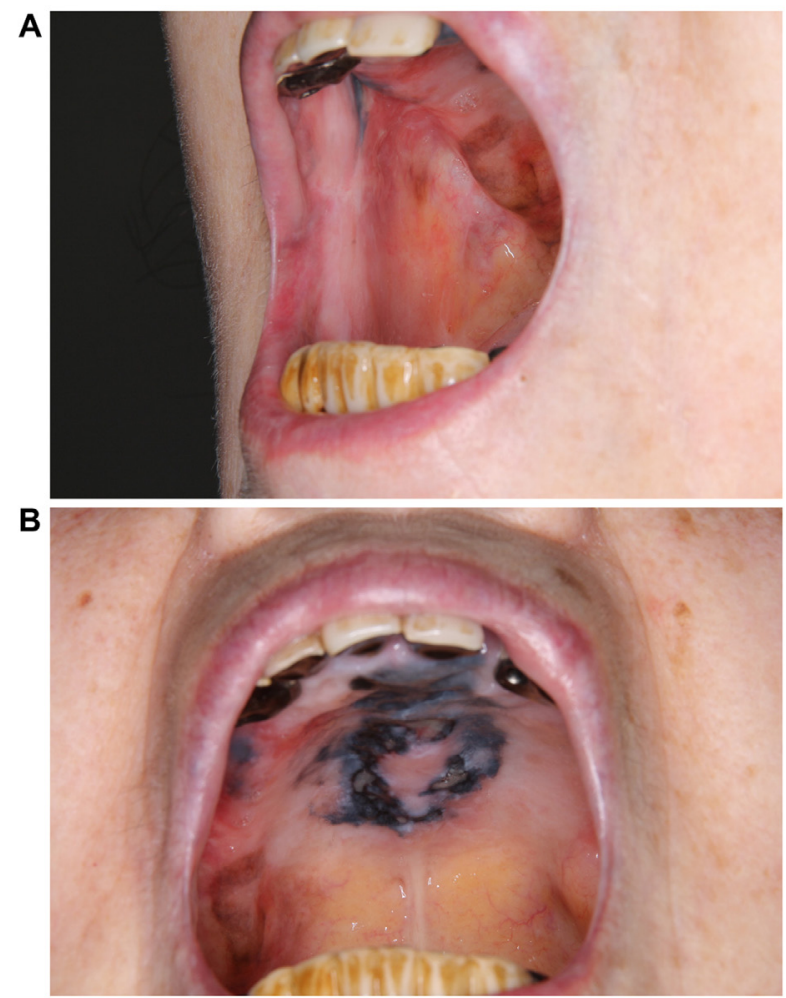

Figure 4. Oral photograph at five months after surgery. (A) The operative wound only left a mild cicatricial contracture and no trismus. The ability of oral intake improved postoperatively. (B) The black lesion on the hard palate had slightly decreased in size with no new lesions. 
The present case report has a limitation: It is a single case report and the disease course success may not be translatable to other cases. Therefore, it cannot be concluded that local treatment should be used in all cases where ICI treatment has failed.

In the future, it may be worthwhile to perform a prospective study or an observational study to evaluate a strategy of adding local treatment to cases in which the general condition is maintained during ICI treatment.

In conclusion, while surgery of the primary head and neck mucosal melanoma with adequate margins is not easy, resecting the tumor for palliation may be beneficial in certain cases, as long as the patient's general physical condition is sufficient to withstand surgery, as was demonstrated in the present study. Tumor burden reduction by surgery, particularly for bulky mass or mass continuing growth despite ICI administration, may bring about a better ICI response or long-term duration of response. The combination of ICIs and surgery may become one of the treatment options in patients with locally advanced mucosal melanoma.

\section{Acknowledgements}

The authors thank Dr Takafumi Okabe (Department of Medical Oncology, Kindai University Nara Hospital, Ikoma, Japan) and Dr Katsuyuki Eto (Department of Otorhinolaryngology, Kindai University Nara Hospital, Ikoma, Japan) for performing patient care.

\section{Funding}

No funding was received.

\section{Availability of data and materials}

The datasets used and/or analyzed during the current study are available from the corresponding author upon reasonable request.

\section{Authors' contributions}

TK, KY and TTak: Conception and design of the study and drafting of the manuscript. TW made the pathological diagnosis. SA, YA and TTam: Management of the patient. TTak and TK checked and approved the authenticity of all the raw data. All authors read and approved the final version of the manuscript.

\section{Ethics approval and consent to participate}

Not applicable.

\section{Patient consent for publication}

Written informed consent for the publication of the present report was obtained from the patient.

\section{Competing interests}

The authors declare that they have no competing interests.

\section{References}

1. Gray-Schopfer V, Wellbrock C and Marais R: Melanoma biology and new targeted therapy. Nature 445: 851-857, 2007.

2. Robert C, Karaszewska B, Schachter J, Rutkowski P, Mackiewicz A, Stroiakovski D, Lichinitser M, Dummer R, Grange F, Mortier L, et al: Improved overall survival in melanoma with combined dabrafenib and trametinib. N Engl J Med 372: 30-39, 2015.

3. Tucker MA: Melanoma epidemiology. Hematol Oncol Clin North Am 23: 383-395, 2009.

4. Robert C, Thomas L, Bondarenko I, O'Day S, Weber J, Garbe C, Lebbe C, Baurain JF, Testori A, Grob JJ, et al: Ipilimumab plus dacarbazine for previously untreated metastatic melanoma. N Engl J Med 364: 2517-2526, 2011.

5. Larkin J, Chiarion-Sileni V, Gonzalez R, Grob JJ, Cowey CL, Lao CD, Schadendorf D, Dummer R, Smylie M, Rutkowski $\mathrm{P}$, et al: Combined nivolumab and ipilimumab or monotherapy in untreated Melanoma. N Engl J Med 373: 23-34, 2015.

6. Robert C, Long GV, Brady B, Dutriaux C, Maio M, Mortier L, Hassel JC, Rutkowski P, McNeil C, Kalinka-Warzocha E, et al: Nivolumab in previously untreated melanoma without BRAF mutation. N Engl J Med 372: 320-330, 2015.

7. D'Angelo SP, Larkin J, Sosman JA, Lebbé C, Brady B, Neyns B, Schmidt H, Hassel JC, Hodi FS, Lorigan P, et al: Efficacy and safety of nivolumab alone or in combination with ipilimumab in patients with mucosal melanoma: A pooled analysis. J Clin Oncol 35: 226-235, 2017.

8. Ozaki Y, Shindoh J, Miura Y, Nakajima H, Oki R, Uchiyama M, Masuda J, Kinowaki K, Kondoh C, Tanabe Y, et al: Serial pseudoprogression of metastatic malignant melanoma in a patient treated with nivolumab: A case report. BMC Cancer 17: 778, 2017.

9. López F, Rodrigo JP, Cardesa A, Triantafyllou A, Devaney KO, Mendenhall WM, Haigentz M Jr, Strojan P, Pellitteri PK, Bradford CR, et al: Update on primary head and neck mucosal melanoma. Head Neck 38: 147-155, 2016.

10. Wolchok JD, Chiarion-Sileni V, Gonzalez R, Rutkowski P, Grob JJ, Cowey CL, Lao CD, Wagstaff J, Schadendorf D, Ferrucci PF, et al: Overall Survival with Combined Nivolumab and Ipilimumab in Advanced Melanoma. N Engl J Med 377: 1345-1356, 2017.

11. Huang AC, Postow MA, Orlowski RJ, Mick R, Bengsch B, Manne S, Xu W, Harmon S, Giles JR, Wenz B, et al: T-cell invigoration to tumour burden ratio associated with anti-PD-1 response. Nature 545: 60-65, 2017.

12. Guisier F, Cousse S, Jeanvoine M, Thiberville L and Salaun M: A rationale for surgical debulking to improve anti-PD1 therapy outcome in non small cell lung cancer. Sci Rep 9: 16902, 2019.

13. Joseph RW, Elassaiss-Schaap J, Kefford R, Hwu WJ, Wolchok JD, Joshua AM, Ribas A, Hodi FS, Hamid O, Robert C, et al: Baseline tumor size is an independent prognostic factor for overall survival in patients with melanoma treated with pembrolizumab. Clin Cancer Res 24: 4960-4967, 2018.

14. Liu Y, Dong Y, Kong L, Shi F, Zhu H and Yu J: Abscopal effect of radiotherapy combined with immune checkpoint inhibitors. J Hematol Oncol 11: 104, 2018.

15. Oronsky B, Larson C, Reid TR and Carter CA: Case series: Abscopal benefit of surgery in 3 immunotherapy-treated patients with unresectable cancer. J Investig Med High Impact Case Rep: Jul 6, 2018 (Epub ahead of print). doi: $10.1177 / 2324709618786319$.

16. Eggermont AMM, Chiarion-Sileni V, Grob JJ, Dummer R, Wolchok JD, Schmidt H, Hamid O, Robert C, Ascierto PA, Richards JM, et al: Prolonged survival in stage III melanoma with ipilimumab adjuvant therapy. N Engl J Med 375: 1845-1855, 2016.

17. Weber J, Mandala M, Del Vecchio M, Gogas HJ, Arance AM, Cowey CL, Dalle S, Schenker M, Chiarion-Sileni V, MarquezRodas I, et al; CheckMate 238 collaborators: Adjuvant nivolumab versus ipilimumab in resected stage III or IV melanoma. N Engl J Med 377: 1824-1835, 2017.

18. Eggermont AMM, Blank CU, Mandala M, Long GV, Atkinson V, Dalle S, Haydon A, Lichinitser M, Khattak A, Carlino MS, et al: adjuvant pembrolizumab versus placebo in resected stage III melanoma. N Engl J Med 378: 1789-1801, 2018. 